\title{
PARP-1 Inhibition Rescues Short Lifespan in Hyperglycemic C. Elegans And Improves GLP-1 Secretion in Human Cells
}

\author{
Qianghua Xia, \#, Sumei Lu", Julian Ostrovsky ${ }^{1}$, Shana E. McCormack ${ }^{2,3,4}$, Marni J. Falk ${ }^{1,3}$, \\ Struan F. A. Grant ${ }^{1,2,3,4, *}$
}

${ }^{1}$ Division of Human Genetics, The Children's Hospital of Philadelphia, Philadelphia, PA 19104, USA. ${ }^{2}$ Division of Endocrinology and Diabetes, The Children's Hospital of Philadelphia, Philadelphia, PA 19104, USA. ${ }^{3}$ Department of Pediatrics, Perelman School of Medicine, University of Pennsylvania, Philadelphia, PA 19104, USA. ${ }^{4}$ Institute of Diabetes, Obesity and Metabolism, Perelman School of Medicine, University of Pennsylvania, Philadelphia, PA 19104, USA

[Received January 5, 2017; Revised February 28, 2017; Accepted February 30, 2017]

\begin{abstract}
TCF7L2 is located at one of the most strongly associated type 2 diabetes loci reported to date. We previously reported that the most abundant member of a specific protein complex to bind across the presumed causal variant at this locus, rs7903146, was poly [ADP-ribose] polymerase type 1 (PARP-1). We analyzed the impact of PARP-1 inhibition on $C$. elegans health in the setting of hyperglycemia and on glucose-stimulated GLP-1 secretion in human intestinal cells. Given that high glucose concentrations progressively shorten the lifespan of $C$. elegans, in part by impacting key well-conserved insulin-modulated signaling pathways, we investigated the effect of PARP-1 inhibition with Olaparib on the lifespan of $C$. elegans nematodes under varying hyperglycemic conditions. Subsequently, we investigated whether Olaparib treatment had any effect on glucose-stimulated GLP-1 secretion in the human NCI-H716 intestinal cell line, a model system for the investigation of enteroendocrine function. Treatment with 100uM Olaparib in nematodes exposed to high concentrations of glucose led to significant lifespan rescue. The beneficial lifespan effect of Olaparib appeared to require both $P A R P$-1 and $T C F 7 L 2$, since treatment had no effect in hyperglycemic conditions in knock-out worm strains for either of these homologs. Further investigation using the NCI-H716 cells revealed that Olaparib significantly enhanced secretion of the incretin, GLP-1, plus the gene expression of TCF7L2, GCG and $P C 1$. These data from studies in both $C$. elegans and a human cell line suggest that PARP-1 inhibition offers a novel therapeutic avenue to treat type 2 diabetes.
\end{abstract}

Key words: TCF7L2, PARP-1, glucose, GLP-1, C. elegans

Type 2 diabetes (T2D) in many cases stems from an agerelated deterioration in insulin signaling and glucose homeostasis. The pathogenesis of T2D is complex and incompletely understood. Caenorhabditis elegans $(C$. elegans) has been used as a model to study diabetes [1], because it has the evolutionary-conserved insulinsignaling pathway that is crucial to both aging and glucose metabolism, making it an informative system in which to study these processes [2].

In this study, we have used this model animal to investigate the functional significance of a finding derived from genome-wide association studies (GWAS), where many loci associated with T2D have been identified [3]. The signal within intron 3 of the TCF7L2 gene on

*Correspondence should be addressed to: Dr. Struan F.A. Grant, Divisions of Human Genetics and Endocrinology, The Children's Hospital of Philadelphia, Philadelphia, PA 19104, USA. Email: grants@ chop.edu. \#These authors contributed equally to this work.

Copyright: ( 2017 Xiao Q et al. This is an open-access article distributed under the terms of the Creative Commons Attribution License, which permits unrestricted use, distribution, and reproduction in any medium, provided the original author and source are credited. 
chromosome 10q25.2 represents one of the strongest genetic associations with T2D risk reported to date, and has been shown to be highly relevant across multiple populations [4, 5]. Furthermore, Bayesian modeling and studies leveraging the haplotypic diversity seen in populations of African ancestry have implicated rs7903146 as the causal SNP at this locus [6].

TCF7L2 (formerly TCF4) is a well-established colorectal cancer gene, encoding a transcription factor that functions as the major effector of the canonical Wnt signaling pathway that plays multiple roles in cell fate determination, survival, proliferation and migration [7]. However, the ways in which TCF7L2 may exert its influence on T2D risk has not yet been mechanistically determined [8]. A key clue is that this transcription factor binds to the promoter region of the proglucagon $(G C G)$ gene [9]. Proglucagon is subject to post-translational processing by prohormone convertase 1 (PC1) to form glucagon-like peptide-1 (GLP-1), which functions as an incretin synthesized in intestinal $L$ cells. As a gut hormone, GLP-1 secretion is regulated by the presence of nutrients in the lumen of the small intestine. GLP-1 has numerous physiological functions, including enhancement of glucose-stimulated insulin secretion, stimulation of $\beta$-cell anti-apoptosis and proliferation, and inhibition of glucagon secretion (resulting from different post-translational processing of proglucagon), food intake and gastric emptying [1]. The anti-diabetic properties of GLP-1 have generated intense interest in the use of this short peptide and its agonists for the treatment of patients with T2D $[10,11]$. As such, one could postulate that TCF7L2 regulates GLP-1, representing one of a number of plausible mechanisms by which the TCF7L2 locus modulates $\mathrm{T} 2 \mathrm{D}$ risk.

In an effort to identify other putative mechanisms by which the TCF7L2 locus impacts T2D, we previously used oligonucleotide pull-down followed by mass spectrometry analysis, to pinpoint specific protein complexes that bind across rs7903146. The most abundant member was poly [ADP-ribose] polymerase type 1 (PARP-1) [12]. Interestingly, a recent study also showed that the rheumatoid arthritis GWAS-implicated risk variant, CCR6DNP, regulates CCR6 via PARP-1[13].

PARP-1 has been shown to play a role in chromatin structure [14]. Chromatin conformation has been shown to differ between alleles for rs7903146 in human pancreatic islets [15]. Interestingly, there have been other reports suggesting that the TCF7L2 and PARP-1 proteins interact [16], and indeed was confirmed in our previous study through the utilization of co-immunoprecipitation [12]. Also, PARP-1 knockout mice have been shown to be protected from induced diabetes [16-18] [19]. Since PARP-1 inhibitors have already been used in humans for oncologic indications, compounds like Olaparib could be used potentially to reduce PARP-1 inhibitory effects on TCF7L2. We hypothesized that Olaparib-mediated PARP-1 inhibition would therefore result in activation of TCF7L2, and yield a net GLP-1 agonist effect.

To test this hypothesis on a whole-organism scale, we first investigated the effect of PARP-1 inhibition with Olaparib on the lifespan of $C$. elegans nematodes under varying hyperglycemic conditions. Subsequently, we investigated whether Olaparib treatment had any effect on glucose-stimulated GLP-1 secretion in a human NCIH716 intestinal cell line, a model system for the investigation of enteroendocrine function. Collectively, these studies suggest that PARP-1 inhibition may offer a novel avenue to ameliorate the negative cellular and animal effects of hyperglycemia.

\section{MATERIALS AND METHODS}

\section{C. elegans strains and maintenance}

We compared the lifespan of $C$. elegans under hyperglycemic conditions $(16 \mathrm{mM}$ glucose or $25 \mathrm{mM}$ glucose) in the presence or absence of Olaparib. The $C$. elegans strains used in the lifespan studies were wild-type N2 Bristol and PARP1 knock-out mutant pme-1(ok988). Both strains were obtained from the Caenorhabditis Genetic Center (CGC, University of Minnesota). All nematodes were cultivated on nematode growth media (NGM) agar plates [20], which utilized living Escherichia coli bacteria OP50 as a food source, and were maintained at $20^{\circ} \mathrm{C}$.

\section{RNAi experiment}

E. coli HT115 (DE3) that contained the pop-1/pL4440 construct (obtained from Source BioScience) and empty RNAi feeding vector pL4440 (generously provided by Silencing Genomes) were used for NGM agar plates containing $1 \mathrm{mM}$ isopropyl- $\beta$-D-thiogalacto-pyranoside (IPTG) and $25 \mathrm{ug} / \mathrm{ml}$ of carbenicillin. The actively growing E. coli were seeded drop- wise (100 ul) onto NGM / IPTG /carbenicillin plates the day before the experiments, covered with aluminum foil, allowed to dry and induced overnight at room temperature. Four conditions, with empty vector pL4440 or pL4440/pop-1 RNAi and with $0.1 \%$ DMSO (vehicle), or $100 \mathrm{uM}$ Olaparib were performed with $16 \mathrm{mM}$ glucose for each group consisting of $n=100$ age-synchronized worms.

\section{Lifespan Assay}

In the lifespan assays, either water or $0.1 \%$ DMSO was used as vehicle. In the hyperglycemia group, a final concentration on NGM agar plates of either $16 \mathrm{mM}$ 
glucose or $25 \mathrm{mM}$ glucose was used, which corresponded to an estimated $\mathrm{HbA} 1 \mathrm{c}$ plasma concentration of $11.7 \%$ or $17.3 \%$ in human diabetic patients. In drug treatment groups, a final concentration on NGM agar plates of 100 uM of the PARP-1 inhibitor, Olaparib (LC Laboratories, AZD-2281) was used with either $16 \mathrm{mM}$ glucose or 25 $\mathrm{mM}$ glucose. To establish the concentration of the drug used in the study, a separate drug survival test was performed using N2 worms with Olaparib at the different concentrations of $0 \mathrm{uM}, 10 \mathrm{uM}$ and $100 \mathrm{uM}$. We further compared $10 \mathrm{uM}$ and $100 \mathrm{uM}$ of Olaparib in the high glucose condition (data not shown). For the majority of the lifespan runs, we did carry out biological repeats (Supplementary Fig. 1).

The experiments were performed on NGM agar on $35 \mathrm{~mm} \times 10 \mathrm{~mm}$ Petri plates. Bacteria were seeded the day before each experiment. 150 ul solutions of each condition were added to the surface of NGM-agar plates inoculated with Escherichia coli bacteria OP50. 100 agesynchronized, day 0 (first day egg laying) young adult worms were spread evenly across five plates and exposed to each condition during the full adult life cycle. Worms were transferred to a new plate daily to prevent mixture of generations and depletion of food. Lifespan data were scored either daily or every other day. Worms that did not respond after repeated gentle stimulus were classified as dead. Worms that crawled away from the plate or harbored internally hatched worms were excluded from the study.

\section{Cell Culture}

The human NCI-H716 cells were obtained from American Type Culture Collection (ATCC, Manassas, VA) and maintained in RPMI-1640 supplemented with $10 \%$ fetal bovine serum as described by ATCC.

\section{GLP-1 ELISA}

NCI-H716 cells were grown in medium with or without 10 uM Olaparib. After 48 hours, supernatants were replaced by PBS containing $100 \mathrm{uM}$ dipeptidyl peptidase IV inhibitor, diprotin A. Cells were subsequently stimulated for 30 minutes with or without $16 \mathrm{mM}$ glucose. GLP-1 was measured by ELISA (EGLP-35K; Millipore, Billerica, MA) according to the manufacturer's instructions.
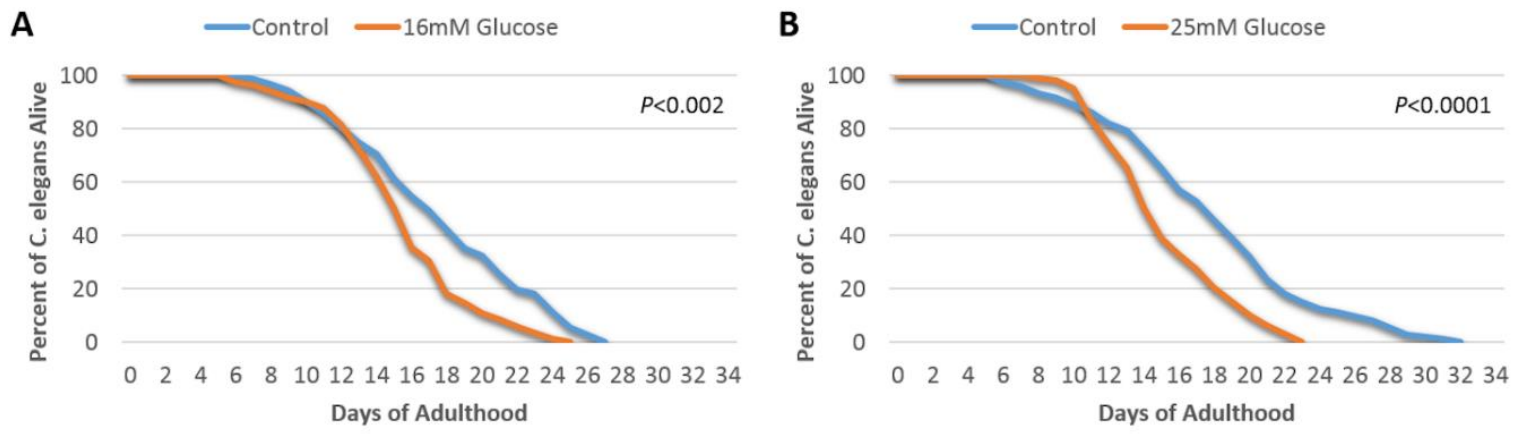

Figure 1. High glucose conditions shorten C. elegans lifespan. Wild-type N2 C. elegans cultured under standard and high glucose conditions and subsequent lifespan assays. (A) Determination of lifespan for N2 worms unexposed or exposed to 16 $\mathrm{mM}$ glucose $(P<0.002$, log-rank test, $\mathrm{n}=100)$. (B) Determination of lifespan for $\mathrm{N} 2$ worms unexposed or exposed to $25 \mathrm{mM}$ glucose $(P<0.0001$, log-rank test, $\mathrm{n}=100)$.

\section{RNA extraction, RT-PCR and real-time PCR}

Total RNA was isolated from the cell pellets using Trizol Reagent (Invitrogen, Carlsbad, CA), according to the manufacturer's protocol, and RNA concentrations were determined by spectrophotometer (NanoDrop ND-1000; Thermo Scientific, Wilmington, DE). Reverse transcription reactions were performed using HighCapacity RNA-to-cDNA ${ }^{\mathrm{TM}}$ Kit (Applied Sciences, Catalog Number: 4387406) according to the manufacturer's protocol. Resultant cDNA was diluted
1:10 and used for quantitative real-time PCR reactions. The primer sequences used for amplification of TCF7L2 were as follows: 5'-GCG GAA AGA CGG CCT CCG CCT CGC-3' and 5'-GGC TTG TCT ACT CTG GAG GCT CCC-3'. The reactions were performed in a total volume of $15 \mu 1$ containing $2 \mu \mathrm{l}$ cDNA, $2 x$ SYBR ${ }^{\circledR}$ Green Universal Master Mix (all real-time PCR reagents were obtained from Applied Biosystems, Foster City, CA). The reactions were run in a 384-well plate in ABI 7900 realtime PCR machine and analyzed with Sequence Detection Systems $7900 \mathrm{HT}$ version 2.4 software (Applied 
Biosystems, Foster City, CA). Briefly, samples were heated to $50^{\circ} \mathrm{C}$ for 2 minutes and then $95^{\circ} \mathrm{C}$ for 10 minutes to allow for DNA polymerase activation followed by 40 cycles of $95^{\circ} \mathrm{C}$ for 15 seconds and $60^{\circ} \mathrm{C}$ for 1 minute to allow for denaturing, annealing, and extension. Data were analyzed using the standard curve method with pooled cDNA serving as the standard. TCF7L2 levels were normalized to GAPDH. The primers used for $G C G$, $P C 1$ and eukaryotic 18S rRNA were purchased from Life Technologies (Cat. \# 4331182, 4331182 and 4453320). Proglucagon and PC1 levels were normalized to eukaryotic 18S rRNA.

\section{Statistical Analysis}

Log-Rank (Mantel-Cox) Test was used for survival analysis and Graph Pad Prism 5 software was used to generate all the lifespan graphs, as previously reported [20]. The two-tailed student's t-test was used to compare expression level between the control and the treated cells. All values were reported as mean $\pm \mathrm{SD}$ and statistical significance defined as $P<0.05$.

\section{RESULTS}

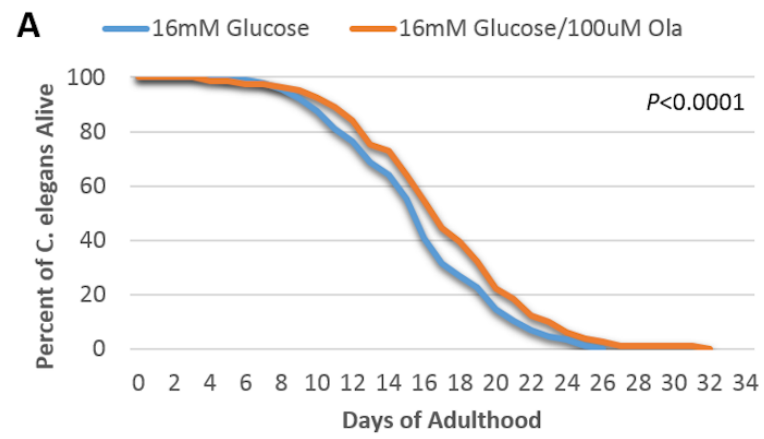

The lifespan of wild-type C. elegans was shortened by high glucose concentrations

We tested the effect of high levels of glucose on the lifespan of $C$. elegans. Wild- type N2 worms were exposed to either $16 \mathrm{mM}$ or $25 \mathrm{mM}$ glucose, which has been shown to produce glucose toxicity in previous studies in C. elegans[1]. As shown in Fig. 1A, the median lifespan of worms exposed to $16 \mathrm{mM}$ glucose was 16 days, with a maximum lifespan of 25 days, compared to a 17-day median lifespan and 27-day maximum lifespan under conditions without high glucose exposure $(P<0.002)$. Furthermore, the higher concentration of glucose $(25 \mathrm{mM})$ showed an even stronger adverse effect on lifespan, where the median lifespan of worms exposed to $25 \mathrm{mM}$ glucose was 15 days and the maximum lifespan was 23 days, which was also significantly shorter than the median and maximum of the concurrent no glucose control condition of 20 days and 30 days, respectively (Fig. 1B, $P<0.0001$ ). These results confirm that high glucose significantly reduce lifespan of wild-type $C$. elegans worms, consistent with results from previous studies [1,21, 22].

Figure 2. The lifespan-shortening effects of high glucose reduced by Olaparib treatment. (A) Determination of lifespan for $\mathrm{N} 2$ worms exposed to $16 \mathrm{mM}$ glucose together with $100 \mathrm{uM}$ or $0 \mu \mathrm{M}$ Olaparib $(P<0.0001, \log -\operatorname{rank}$ test, $\mathrm{n}=100)$. (B) Determination of lifespan for $\mathrm{N} 2$ worms exposed to $25 \mathrm{mM}$ glucose together with $100 \mathrm{uM}$ or $0 \mu \mathrm{M}$ Olaparib $(P<0.05, \log$-rank test, $\mathrm{n}=100)$.

Treatment with Olaparib improves shortened lifespan in hyperglycemic conditions in wild-type, but not PARP1 homologue (pme-1) knockout, C. elegans

We observed that the lifespan of wild-type N2 C. elegans was significantly extended by the addition of $100 \mathrm{uM}$ Olaparib in $16 \mathrm{mM}$ glucose (Fig. $2 \mathrm{~A}, P<0.0001$ ). We also observed a benefit of $100 \mathrm{uM}$ Olarparib in $25 \mathrm{mM}$ glucose (Fig. 2B, $P<0.05$ ). These results indicate a beneficial effect of PARP-1 inhibition by Olaparib on lifespan under

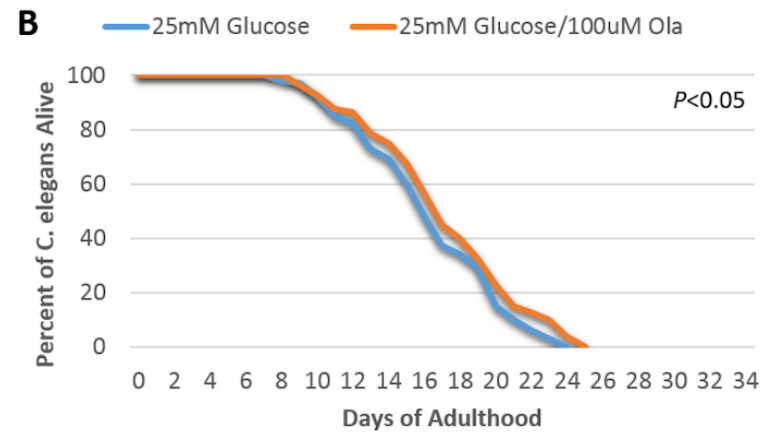

conditions of glucose toxicity. However, upon repeating these experiments in the PARP-1 knock-out mutant strain, pme-1, (mutation shown in Supplementary Fig. 2), Olaparib yielded no incremental benefit on lifespan in either $16 \mathrm{mM}$ (Fig. 3A) or $25 \mathrm{mM}$ (Fig. 3B) glucose. These data suggest that the observed beneficial effect of Olaparib on rescuing hyperglycemia-induced short lifespan may be target-specific and directly depends on the PARP-1 signaling pathway. 

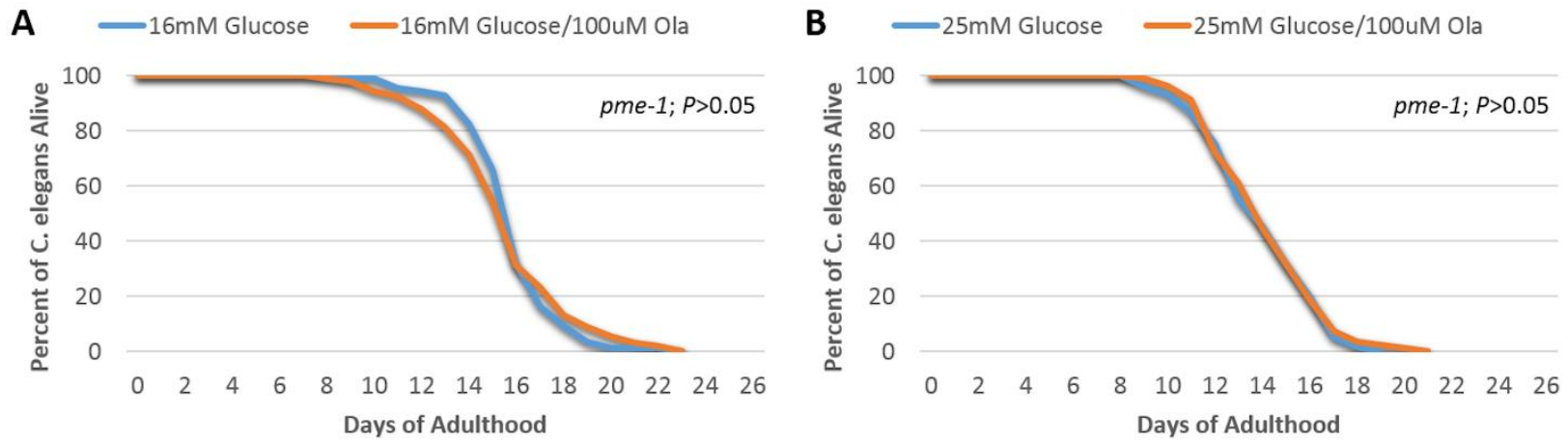

Figure 3. The beneficial effect of Olaparib treatment on C. elegans lifespan in the setting of high glucose was target-specific and dependent on the PARP-1 related signaling pathway. (A) Determination of lifespan for $P A R P-1$ homolog, pme-1, worms exposed to $16 \mathrm{mM}$ glucose together with $100 \mu \mathrm{M}$ or without Olaparib $(P>0.05, \log$-rank test, $\mathrm{n}=100)$. (B) Determination of lifespan for $p m e-1$ worms exposed to $25 \mathrm{mM}$ glucose together with $100 \mu \mathrm{M}$ or $0 \mu \mathrm{M}$ Olaparib $(P>0.05$, log-rank test, $\mathrm{n}=100)$.

TCF7L2 (pop-1) is required for the beneficial effect of Olaparib on lifespan in C. elegans

We investigated whether the effect of PARP-1 inhibition on C. elegans lifespan could be mediated by TCF7L2. To examine this possibility, we further compared the lifespan of knock-down worms for the TCF7L2 homolog, pop-1 (Supplementary Figure 3), under hyperglycemic conditions with or without Olaparib. Both the pop- 1 RNAi worms and wild-type N2 control worms were exposed to
$16 \mathrm{mM}$ glucose with or without $100 \mathrm{uM}$ Olaparib. As expected, treatment with Olaparib significantly prolonged lifespan of the worms in the wild-type worms exposed to $16 \mathrm{mM}$ glucose (Fig. 4A, $P<0.03$ ). In contrast, treatment with Olaparib did not have a significant effect on lifespan of the worms in the pop-1 RNAi group (Fig. 4B). These results suggest that pop-1 (TCF7L2) is required for the regulation of $C$. elegans lifespan by a PARP-1 inhibitor in the presence of high glucose.
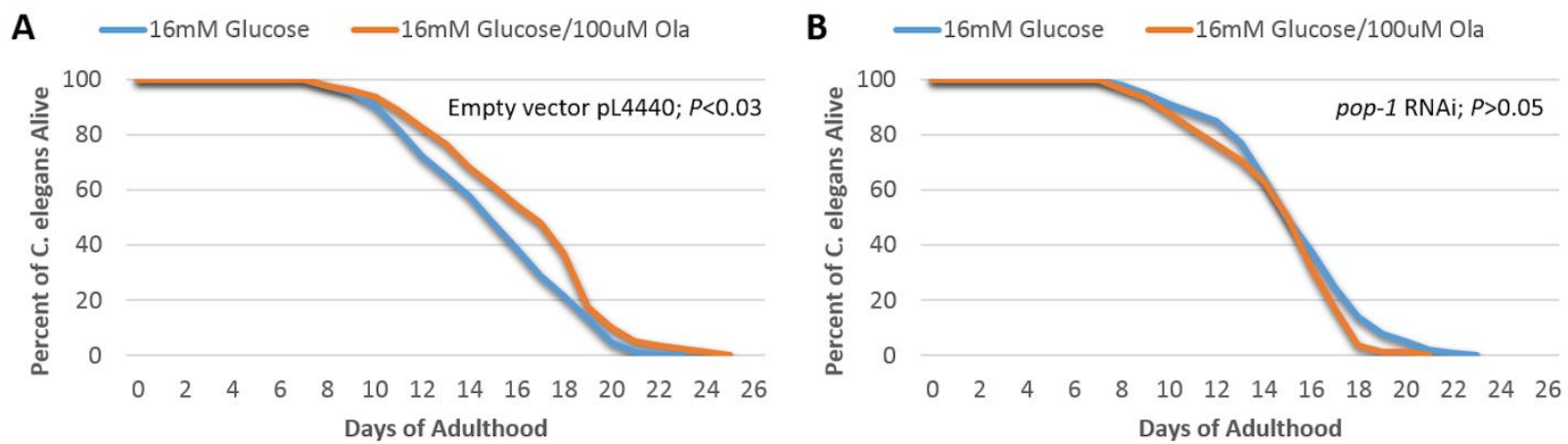

Figure 4. TCF 7L2 homolog, pop-1, is required for the beneficial effect in the setting of high glucose of Olaparib treatment on lifespan. (A) Determination of lifespan for N2 worms fed with HT115 feeding strain containing the pL4440 empty vector and exposed to $16 \mathrm{mM}$ glucose with $100 \mu \mathrm{M}$ or $0 \mathrm{uM}$ Olaparib $(P<0.03$, log-rank test, $\mathrm{n}=100)$. (B) Determination of lifespan for $\mathrm{N} 2$ worms fed with HT115 feeding strain containing the pL4440 pL4440/pop-1 RNAi and exposed to $16 \mathrm{mM}$ glucose with $100 \mathrm{uM}$ or $0 \mu \mathrm{M}$ Olaparib $(P>0.05$, log-rank test, $\mathrm{n}=100)$. 


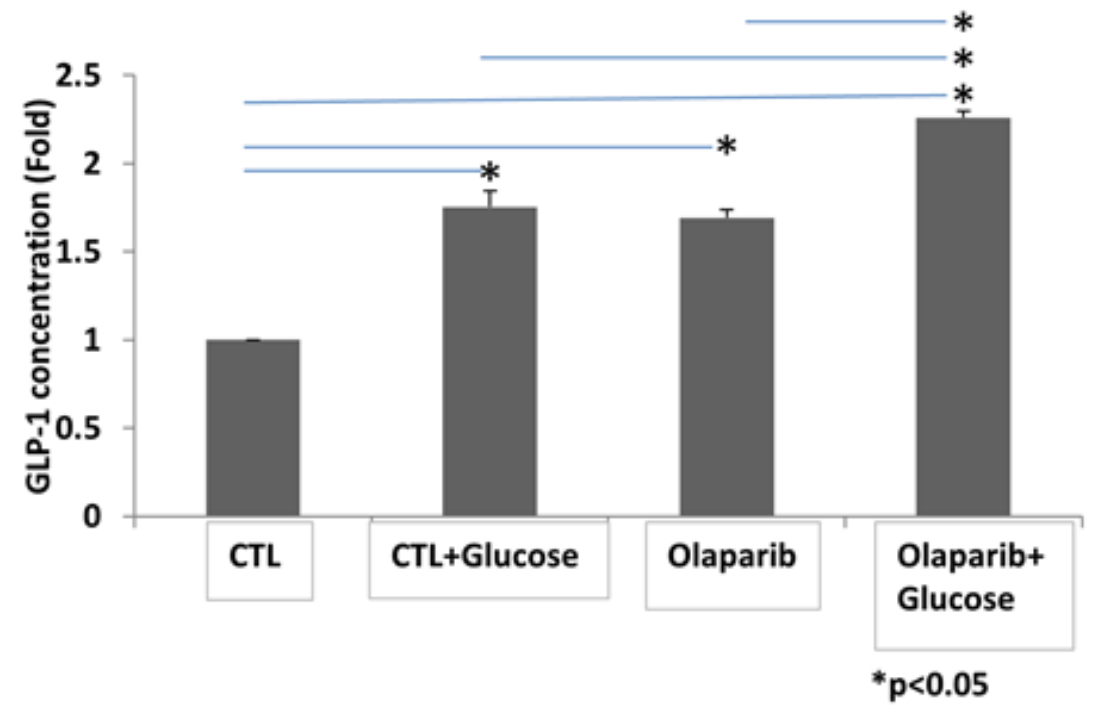

Figure 5. Olaparib enhances promotes GLP-1 secretion in NCI-H716 cells. Cells were stimulated for 30 minutes with or without $16 \mathrm{mM}$ glucose. GLP-1 was measured by ELISA. Bars represent the mean of three independent experiments normalized to the control. Error bars indicate standard deviation. Statistical analyses were performed by two-tailed Student's t-test and significance is denoted by asterisks where $* P<0.05$.

\section{Olaparib treatment increases GLP-1 secretion in human NCI-H716 enteroendocrine intestinal cells}

Insulin signaling pathways have been implicated in aging and longevity in both worms and mammals [23, 24]. Abnormal insulin homeostasis may underlie the glucosemediated decrease in lifespan we observed in C. elegans, and the beneficial effect of PARP-1 inhibition may result from its downstream effects on insulin production and action. To examine the translational potential of using a PARP-1 inhibitor to treat the sequelae of hyperglycemia in humans, we used the NCI-H716 enteroendocrine cell line that is widely used to study human GLP-1-secretion, an important modulator of insulin production [25]. As shown in Fig. 5, stimulation of NCI-H716 cells with glucose increased GLP-1 release into the medium $(\mathrm{p}<0.05)$. Interestingly, Olaparib treatment alone also increased GLP-1 secretion significantly as compared with untreated cells $(51.1 \%$ average increase; $P<0.05)$. Most importantly, levels of active GLP-1 were further increased by combining glucose plus Olaparib as compared with either glucose or Olaparib alone $(26.0 \%$ and $29.4 \%$ average increase, respectively; $P<0.05)$. The combination of glucose and Olaparib increased GLP-1 up to 2.2 fold $(P<0.05)$. These data suggest that Olaparib induces GLP1 secretion both in the absence of glucose, and to a greater degree, in its presence.

\section{Olaparib increases enteroendocrine intestinal cell expression of TCF7L2, GCG and PC1}

Given the known interaction between TCF7L2 and the promoter region of the GCG gene [9], we hypothesized that PARP-1 interplay with TCF7L2 has an inhibitory effect on TCF7L2 expression and its downstream stimulation of $G C G$ and $P C l$ expression. Using quantitative real-time PCR, we compared the expression levels of TCF $2 L 2$ with and without Olaparib treatment for 48 hours in NCI-H716 cells. Our results showed that Olaparib significantly increased TCF7L2 expression $(P<0.05)$ (Fig. 6A). Since we observed that GLP-1 secretion is upregulated by Olaparib treatment, we postulated that both $G C G$ and $P C 1$ expression could also be impacted by PARP-1 inhibition. Indeed, quantitative real time-PCR gene expression results demonstrated that $G C G$ and $P C l$ expression were significantly increased by up to two-fold with Olaparib treatment (Fig. 6B and 6C, $P<0.01)$. These findings support our hypothesis by showing that PARP-1 inhibition with Olaparib reverses the inhibitory effects of PARP-1 on TCF7L2, with subsequent increases in TCF $2 L 2$ and downstream $G C G$ and $P C 1$ gene expression, and net positive effects on GLP-1 production. 

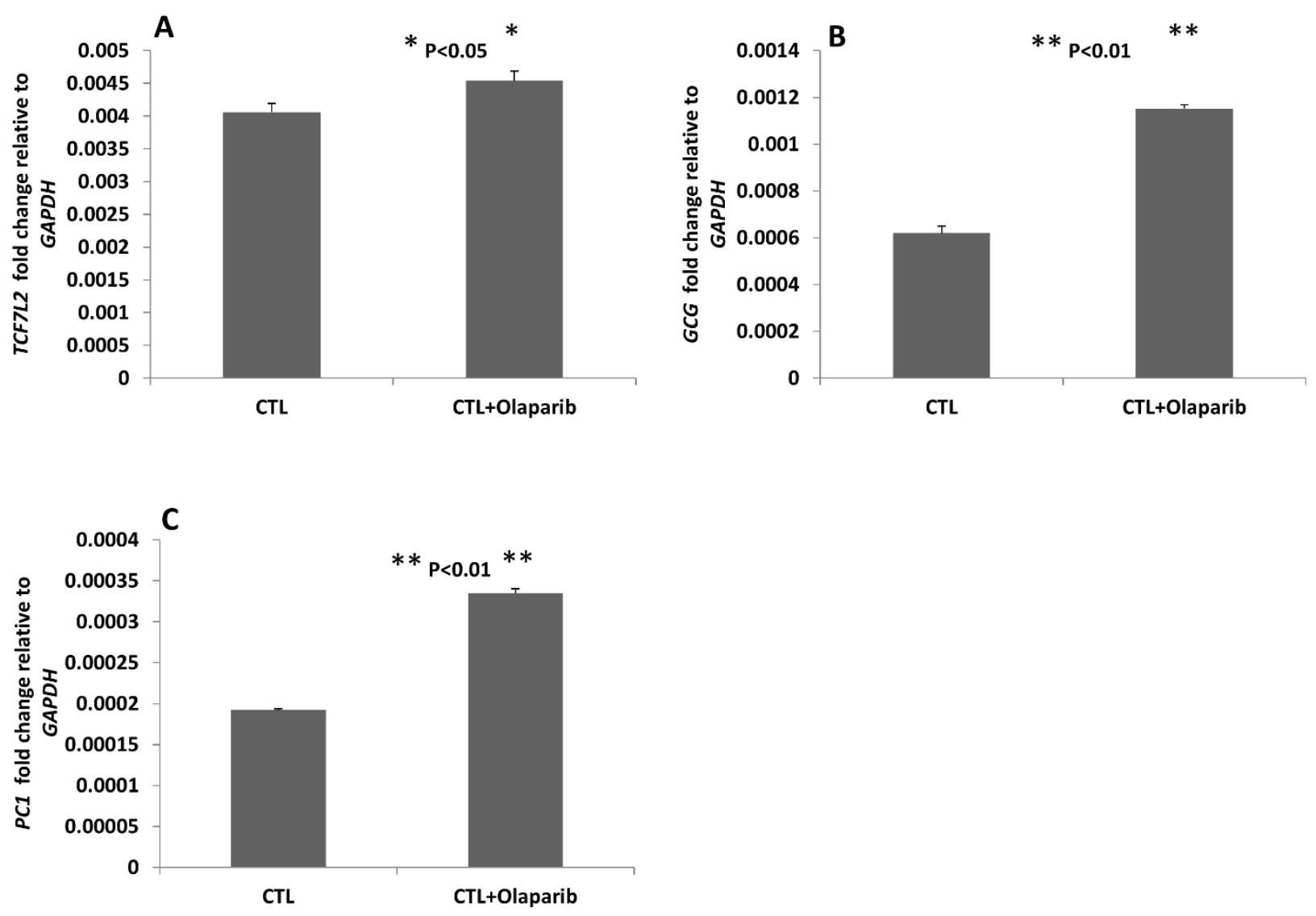

Figure 6. Olaparib treatment of endoendocrine intestinal cells increases expression of (A)TCF7L2, (B) GCG and (C) PC1. Relative levels of individual gene expression in the control and the Olaparib treatment groups determined by quantitative PCR with GAPDH normalization. Values are the mean of three independent experiments. Error bars indicate standard deviation. Statistical analyses were performed by two-tailed Student's t-test and significance is denoted by asterisks where $* P<0.05$ or $* * P<0.01$.

\section{DISCUSSION}

Our prior discovery that the genomic region harboring the presumed T2D causal variant at the TCF7L2 locus, namely rs7903146, binds a protein complex that includes PARP-1 prompted us to functionally evaluate whether PARP-1 inhibition may have anti-diabetic effects.

Although previously implicated in the mediation of diabetes microvascular complications[26], PARP-1 has not been an obvious candidate in the direct pathogenesis of T2D, with a known role principally in DNA damage repair through repair of single-strand $[27,28]$ and doublestrand [29] breaks. A disease role for PARP-1 has been largely the domain of oncology studies, with several PARP-1 inhibitors pursued through clinical trials for various cancers. In particular, Olaparib has been tested in patients with BRCA-mutated tumors including breast, ovarian and colorectal cancer in phase II clinical trials [30, 31], with well tolerated side-effects [32]. Interestingly, however, evidence exists for a protective effect of PARP1 inhibition on the pancreatic $\beta$ cell, albeit via a mechanism that has not yet been fully elucidated. Specifically, pre-treatment with the PARP-1 inhibitors nicotinamide and 3 -aminobenzamide have been shown to reverse a streptozocin-induced decrease in proinsulin synthesis in rats [33]. Furthermore, other gene products related to DNA damage repair have been implicated in diabetes, most recently XRCC4 [34].

Many key metabolic signaling pathways, including insulin, have homologues identified in C. elegans [35-39]. 
Given that PARP-1, as a potential drug target, is also conserved between $C$. elegans and humans, we investigated the mechanism by which PARP-1 inhibition could influence the negative effects of hyperglycemia in the $C$. elegans model animal. It is well-established that increased glucose levels shorten C. elegans lifespan, with induction of reactive oxygen species formation and subsequent cellular and systemic damage [1, 21, 22]. Our baseline results confirmed that feeding wild-type $C$. elegans with moderately $(16 \mathrm{mM})$ or severely $(25 \mathrm{mM})$ high glucose diets significantly reduced their lifespan. Conversely, reduced glucose metabolism has been shown to extend lifespan [40, 41], with disruption of certain glycolytic genes also extending lifespan in nematodes [42, 43]. We discovered that PARP-1 inhibition rescued the shortened lifespan of $C$. elegans under hyperglycemic stress, where this pharmacological effect was PARP-1 specific since Olaparib treatment had no effect on mutants for the PARP-1 homolog, pme-1. Beneficial effects of Olaparib on lifespan were also ablated by feeding RNAi knock-down of the TCF7L2 homolog, pop-1, suggesting that TCF7L2 is required to gain therapeutic benefit from PARP-1 inhibition in the context of hyperglycemia. The mechanism by which pop-1 mediates this effect remains unclear, with further work warranted to elucidate the precise role of the TCF7L2/pop-1 homolog in rescuing hyperglycemia-induced toxicity by PARP-1 inhibition. However, our results point to a potential utility of the anticancer drug class of PARP-1 inhibitors in the therapeutic context of primary metabolic traits.
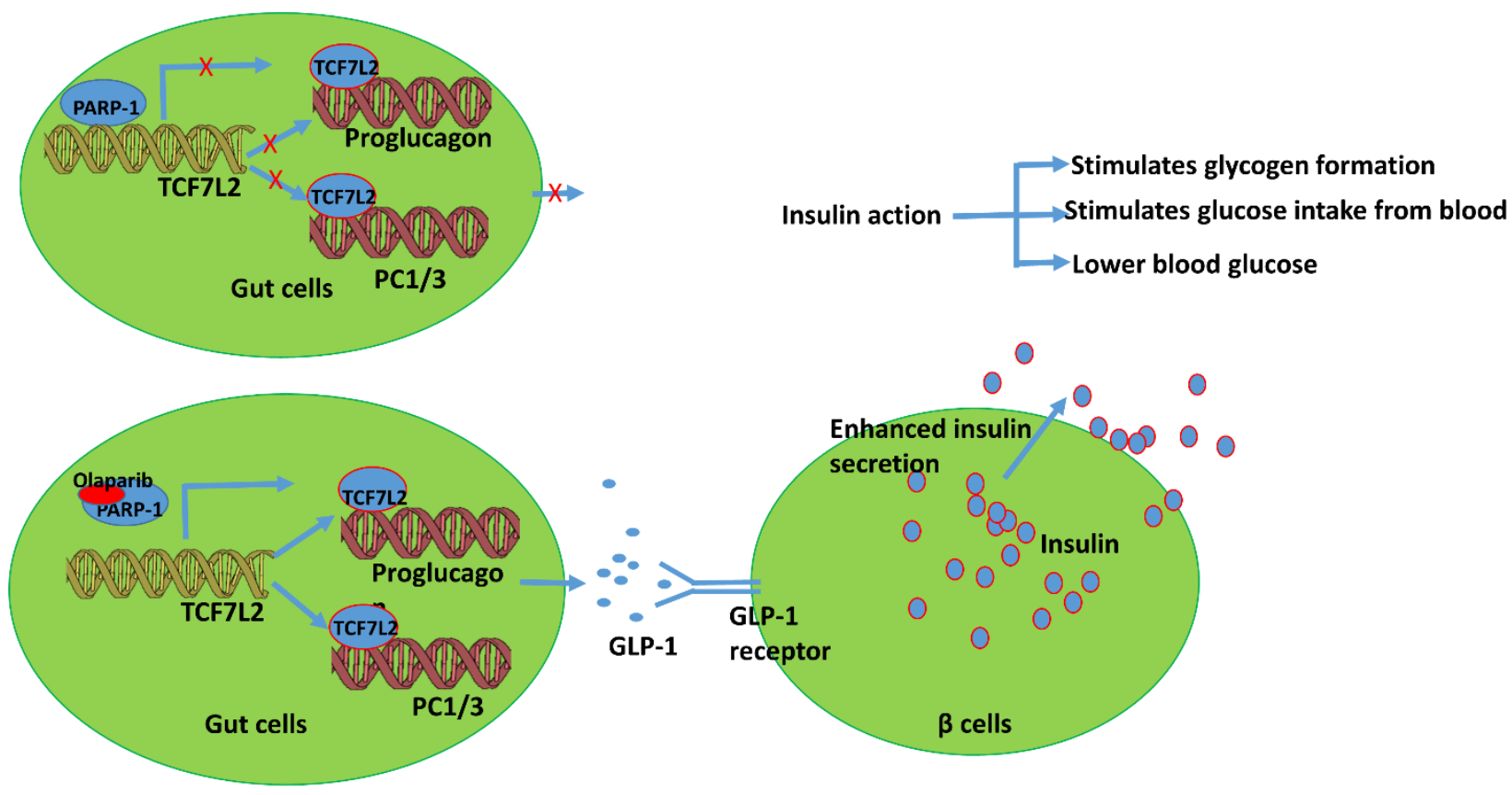

Figure 7. Integrated model to define the role of PARP-1 inhibition in the type 2 diabetes related pathway. Olaparib treatment increases the expression of $T C F 7 L 2, G C G$ and $P C 1$, thereby enhancing GLP-1 secretion in the endoendocrine L type gastrointestinal cells and subsequently promoting insulin secretion in the pancreatic $\beta$-cell.

Given these results, we used a human cellular model relevant to diabetes mellitus to investigate the mechanistic relationship between TCF7L2 and GLP-1. GLP-1, which plays a crucial role in insulin secretion, is produced in intestinal cells from selective cleavage of the transcription product encoded by $G C G$. Specifically, higher levels of prohormone convertase 1 (encoded by $P C 1$ ) in L cells contribute to the processing of proglucagon to liberate GLP-1 [44]. Indeed, a great deal of effort has been made to improve GLP-1 action for the development of new therapeutic approaches to treat T2D [45, 46]. Since it is known that expression of $G C G$ is driven by TCF7L2 in intestinal endocrine L-cells $[9,47]$ and the NCI-H716 cell line offers a unique model to study the cellular mechanism of GLP-1 secretion in humans [25], we tested the efficacy of a PARP-1 inhibition strategy under hyperglycemic conditions in this cellular model. Collectively, our data suggest an integrated model to support the role of PARP- 
1 inhibition in T2D-related cellular pathophysiology (Fig. 7). We propose that Olaparib treatment increases intestinal L cell expression of key genes TCF7L2, GCG and $P C 1$, that led to enhanced GLP-1 release in to the gastrointestinal track, consequently augmenting glucosestimulated insulin secretion in the pancreatic $\beta$-cell.

We acknowledge that some debate has surrounded the issue of what protein factors actually bind across the key SNP harbored within TCF7L2. One study suggested HMGB1 [48], while another implicated FOXA2 [49]. Although there is a possibility that all these factors play a role in transcriptional control at this genomic region, our current functional data presented here adds further support to our prior findings that PARP-1 is a physiologically important binding partner of TCF7L2 [12]. In addition to support for this factor coming from the rheumatoid arthritis risk variant CCR6DNP regulating CCR6 via PARP-1[13], it is interesting to note that a recent age at menopause GWAS linked the loci found in that effort to DNA damage response genes[50].

In addition to PARP-1, we recognize that Olaparib can also influence the expression of PARP-2. However, given that we see an influence of Olaparib on lifespan and that independently knocking out pme-1 (the homolog of $P A R P-1$ specifically) has a comparable impact, one must conclude that PARP-1 is playing a role in this observation; however, one cannot rule an influence by or on PARP-2 to some degree in this current dataset.

It should be noted that we previously reported an additional gene at the locus, namely ACSL5, together with providing further support for TCF7L2 [51]. There is now an abundance of studies lending a high degree of support for TCF7L2 itself playing a role in T2D pathogenesis at this locus in various tissue contexts. Combined with multiple lines of evidence that the TCF7L2 protein product has a physical interplay with PARP-1, we were motivated us to investigate this relationship further. On the other hand, the role of ACSL5 in the context of type 2 diabetes pathogenesis still needs to be fully elucidated, including how it relates to PARP-1, before one can meaningfully embark on a similar study for that particular factor.

Taken together, these observations suggest that PARP-1 inhibition increases TCF7L2-mediated GLP-1 production. Therefore, pharmacologic PARP-1 inhibition may represent an avenue for therapeutic intervention for T2D. The classical dipeptidyl peptidase-4 (DPP-IV) inhibitors that protect the GLP-1 peptide from degradation have been used successfully as therapeutic reagents for T2D [52]. Our studies suggest a novel mechanism by which the PARP-1 inhibitor, Olaparib, effectively enhances GLP-1 activity in the setting of hyperglycemia and could represent an alternative therapeutic avenue to that of DPP-IV inhibition for T2D.

\section{Acknowledgements}

The work was financially supported by Pennsylvania Department of Health award SAP4100062202 and the Daniel B. Burke Endowed Chair for Diabetes Research.

\section{References}

[1] Lee SJ, Murphy CT, Kenyon C (2009). Glucose shortens the life span of C. elegans by downregulating DAF16/FOXO activity and aquaporin gene expression. Cell Metab, 10:379-391.

[2] Finch CE, Ruvkun G (2001). The genetics of aging. Annu Rev Genomics Hum Genet, 2:435-462.

[3] Visscher PM, Brown MA, McCarthy MI, Yang J (2012). Five years of GWAS discovery. Am J Hum Genet, 90:724.

[4] Grant SF, Thorleifsson G, Reynisdottir I, Benediktsson R, Manolescu A, Sainz J, et al. (2006). Variant of transcription factor 7-like 2 (TCF7L2) gene confers risk of type 2 diabetes. Nat Genet, 38:320-323.

[5] DIAbetes Genetics Replication And Meta-analysis (DIAGRAM) Consortium (2014). Genome-wide transancestry meta-analysis provides insight into the genetic architecture of type 2 diabetes susceptibility. Nat Genet, 46:234-244.

[6] Wellcome Trust Case Control C, Maller JB, McVean G, Byrnes J, Vukcevic D, Palin K, et al. (2012). Bayesian refinement of association signals for 14 loci in 3 common diseases. Nat Genet, 44:1294-1301.

[7] MacDonald BT, Tamai K, He X (2009). Wnt/betacatenin signaling: components, mechanisms, and diseases. Dev Cell, 17:9-26.

[8] Pearson ER (2009). Translating TCF7L2: from gene to function. Diabetologia, 52:1227-1230.

[9] Ni Z, Anini Y, Fang X, Mills G, Brubaker PL, Jin T (2003). Transcriptional activation of the proglucagon gene by lithium and beta-catenin in intestinal endocrine L cells. J Biol Chem, 278:1380-1387.

[10] Langley AK, Suffoletta TJ, Jennings HR (2007). Dipeptidyl peptidase IV inhibitors and the incretin system in type 2 diabetes mellitus. Pharmacotherapy, 27:1163-1180.

[11] Drucker DJ, Nauck MA (2006). The incretin system: glucagon-like peptide-1 receptor agonists and dipeptidyl peptidase-4 inhibitors in type 2 diabetes. Lancet, 368:1696-1705.

[12] Xia Q, Deliard S, Yuan CX, Johnson ME, Grant SF (2015). Characterization of the transcriptional machinery bound across the widely presumed type 2 diabetes causal variant, rs7903146, within TCF7L2. Eur J Hum Genet, 23:103-109.

[13] Li G, Cunin P, Wu D, Diogo D, Yang Y, Okada Y, et al. (2016). The Rheumatoid Arthritis Risk Variant CCR6DNP Regulates CCR6 via PARP-1. PLoS Genet, 12:e1006292.

[14] Krishnakumar R, Kraus WL (2010). PARP-1 regulates chromatin structure and transcription through a KDM5B-dependent pathway. Mol Cell, 39:736-749. 
[15] Gong L, Liu FQ, Wang Y, Hou XG, Zhang W, Qin WD, et al. (2012). Poly (ADP-ribose) transferase/polymerase1-deficient mice resistant to age-dependent decrease in beta-cell proliferation. Mol Med, 18:816-824.

[16] Idogawa M, Yamada T, Honda K, Sato S, Imai K, Hirohashi S (2005). Poly(ADP-ribose) polymerase-1 is a component of the oncogenic T-cell factor-4/beta-catenin complex. Gastroenterology, 128:1919-1936.

[17] Idogawa M, Masutani M, Shitashige M, Honda K, Tokino T, Shinomura Y, et al. (2007). Ku70 and poly(ADP-ribose) polymerase-1 competitively regulate beta-catenin and T-cell factor-4-mediated gene transactivation: possible linkage of DNA damage recognition and Wnt signaling. Cancer Res, 67:911-918.

[18] Pieper AA, Brat DJ, Krug DK, Watkins CC, Gupta A, Blackshaw S, et al. (1999). Poly(ADP-ribose) polymerase-deficient mice are protected from streptozotocin-induced diabetes. Proc Natl Acad Sci U S A, 96:3059-3064.

[19] Li B, Luo C, Chowdhury S, Gao ZH, Liu JL (2013). Parp1 deficient mice are protected from streptozotocininduced diabetes but not caerulein-induced pancreatitis, independent of the induction of Reg family genes. Regul Pept, 186:83-91.

[20] Dingley S, Polyak E, Lightfoot R, Ostrovsky J, Rao M, Greco T, et al. (2010). Mitochondrial respiratory chain dysfunction variably increases oxidant stress in Caenorhabditis elegans. Mitochondrion, 10:125-136.

[21] Schulz TJ, Zarse K, Voigt A, Urban N, Birringer M, Ristow M (2007). Glucose restriction extends Caenorhabditis elegans life span by inducing mitochondrial respiration and increasing oxidative stress. Cell Metab, 6:280-293.

[22] Schlotterer A, Kukudov G, Bozorgmehr F, Hutter H, Du $\mathrm{X}$, Oikonomou D, et al. (2009). C. elegans as model for the study of high glucose- mediated life span reduction. Diabetes, 58:2450-2456.

[23] Kenyon CJ (2010). The genetics of ageing. Nature, 464:504-512.

[24] van Heemst D, Beekman M, Mooijaart SP, Heijmans BT, Brandt BW, Zwaan BJ, et al. (2005). Reduced insulin/IGF-1 signalling and human longevity. Aging Cell, 4:79-85.

[25] Reimer RA, Darimont C, Gremlich S, Nicolas-Metral V, Ruegg UT, Mace K (2001). A human cellular model for studying the regulation of glucagon-like peptide-1 secretion. Endocrinology, 142:4522-4528.

[26] Du X, Matsumura T, Edelstein D, Rossetti L, Zsengeller Z, Szabo C, et al. (2003). Inhibition of GAPDH activity by poly(ADP-ribose) polymerase activates three major pathways of hyperglycemic damage in endothelial cells. J Clin Invest, 112:1049-1057.

[27] Caldecott KW (2007). Mammalian single-strand break repair: mechanisms and links with chromatin. DNA Repair (Amst), 6:443-453.

[28] Woodhouse BC, Dianov GL (2008). Poly ADP-ribose polymerase-1: an international molecule of mystery. DNA Repair (Amst), 7:1077-1086.
[29] Rouleau M, Patel A, Hendzel MJ, Kaufmann SH, Poirier GG (2010). PARP inhibition: PARP1 and beyond. Nat Rev Cancer, 10:293-301.

[30] Marchetti C, Imperiale L, Gasparri ML, Palaia I, Pignata S, Boni T, et al. (2012). Olaparib, PARP1 inhibitor in ovarian cancer. Expert Opin Investig Drugs, 21:15751584.

[31] Glendenning J, Tutt A (2011). PARP inhibitors--current status and the walk towards early breast cancer. Breast, 20 Suppl 3:S12-19.

[32] Ledermann J, Harter P, Gourley C, Friedlander M, Vergote I, Rustin G, et al. (2012). Olaparib maintenance therapy in platinum-sensitive relapsed ovarian cancer. $\mathrm{N}$ Engl J Med, 366:1382-1392.

[33] Uchigata Y, Yamamoto H, Nagai H, Okamoto H (1983). Effect of poly(ADP-ribose) synthetase inhibitor administration to rats before and after injection of alloxan and streptozotocin on islet proinsulin synthesis. Diabetes, 32:316-318.

[34] Dooley J, Tian L, Schonefeldt S, Delghingaro-Augusto V, Garcia-Perez JE, Pasciuto E, et al. (2016). Genetic predisposition for beta cell fragility underlies type 1 and type 2 diabetes. Nat Genet, 48:519-527.

[35] Consortium CeS (1998). Genome sequence of the nematode C. elegans: a platform for investigating biology. Science, 282:2012-2018.

[36] Sonnhammer EL, Durbin R (1997). Analysis of protein domain families in Caenorhabditis elegans. Genomics, 46:200-216.

[37] Lai CH, Chou CY, Ch'ang LY, Liu CS, Lin W (2000). Identification of novel human genes evolutionarily conserved in Caenorhabditis elegans by comparative proteomics. Genome Res, 10:703-713.

[38] Kuwabara PE, O'Neil N (2001). The use of functional genomics in $\mathrm{C}$. elegans for studying human development and disease. J Inherit Metab Dis, 24:127-138.

[39] Harris TW, Chen N, Cunningham F, Tello-Ruiz M, Antoshechkin I, Bastiani C, et al. (2004). WormBase: a multi-species resource for nematode biology and genomics. Nucleic Acids Res, 32:D411-417.

[40] Yokoyama K, Fukumoto K, Murakami T, Harada S, Hosono R, Wadhwa R, et al. (2002). Extended longevity of Caenorhabditis elegans by knocking in extra copies of hsp70F, a homolog of mot-2 (mortalin)/mthsp70/Grp75. FEBS Lett, 516:53-57.

[41] Urban N, Tsitsipatis D, Gille A, Hamann I, Hou X, Klotz LO (2014). Modulation of cellular thiol status affects FoxO activity and life span. Free Radic Biol Med, 75 Suppl 1:S53.

[42] Hamilton B, Dong Y, Shindo M, Liu W, Odell I, Ruvkun G, et al. (2005). A systematic RNAi screen for longevity genes in C. elegans. Genes Dev, 19:1544-1555.

[43] Lee SS, Lee RY, Fraser AG, Kamath RS, Ahringer J, Ruvkun G (2003). A systematic RNAi screen identifies a critical role for mitochondria in $\mathrm{C}$. elegans longevity. Nat Genet, 33:40-48.

[44] Scopsi L, Gullo M, Rilke F, Martin S, Steiner DF (1995). Proprotein convertases (PC1/PC3 and PC2) in normal and neoplastic human tissues: their use as markers of 
neuroendocrine differentiation. J Clin Endocrinol Metab, 80:294-301.

[45] Donnelly D (2012). The structure and function of the glucagon-like peptide-1 receptor and its ligands. Br J Pharmacol, 166:27-41.

[46] Ahren B (2011). GLP-1 for type 2 diabetes. Exp Cell Res, 317:1239-1245.

[47] Yi F, Brubaker PL, Jin T (2005). TCF-4 mediates cell type-specific regulation of proglucagon gene expression by beta-catenin and glycogen synthase kinase-3beta. J Biol Chem, 280:1457-1464.

[48] Zhou Y, Oskolkov N, Shcherbina L, Ratti J, Kock KH, Su J, et al. (2016). HMGB1 binds to the rs7903146 locus in TCF7L2 in human pancreatic islets. Mol Cell Endocrinol, 430:138-145.

[49] Gaulton KJ, Ferreira T, Lee Y, Raimondo A, Magi R, Reschen ME, et al. (2015). Genetic fine mapping and genomic annotation defines causal mechanisms at type 2 diabetes susceptibility loci. Nat Genet, 47:1415-1425.

[50] Day FR, Ruth KS, Thompson DJ, Lunetta KL, Pervjakova N, Chasman DI, et al. (2015). Large-scale genomic analyses link reproductive aging to hypothalamic signaling, breast cancer susceptibility and BRCA1-mediated DNA repair. Nat Genet, 47:12941303.

[51] Xia Q, Chesi A, Manduchi E, Johnston BT, Lu S, Leonard ME, et al. (2016). The type 2 diabetes presumed causal variant within TCF7L2 resides in an element that controls the expression of ACSL5. Diabetologia.

[52] Tuduri E, Lopez M, Dieguez C, Nadal A, Nogueiras R (2016). Glucagon-Like Peptide 1 Analogs and their Effects on Pancreatic Islets. Trends Endocrinol Metab, 27:304-318. 

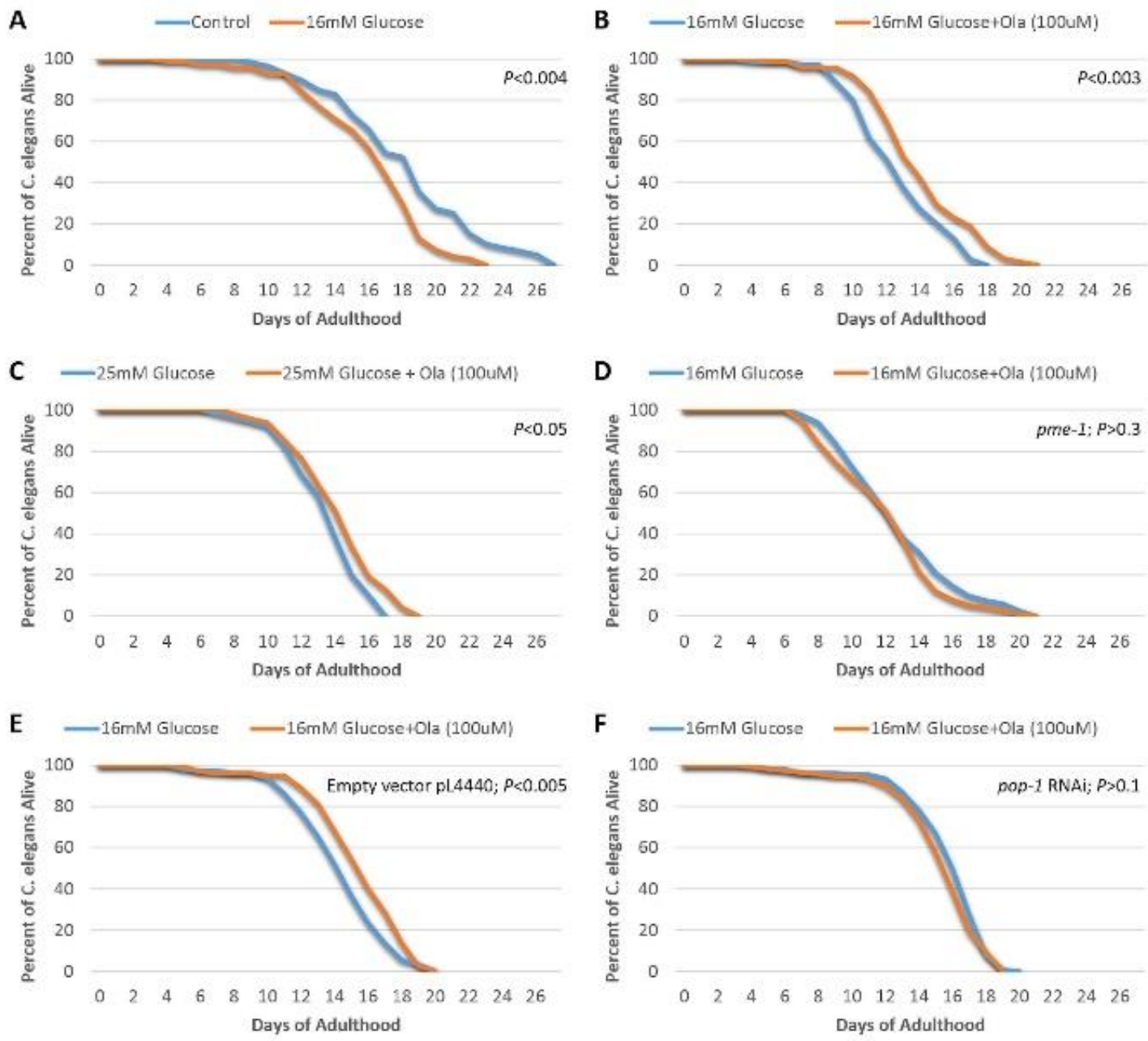

Supplementary Figure 1. Biological replicates of lifespan assays. A) Biological replicate of Figure 1A: High glucose conditions shorten C. elegans lifespan. B) Biological replicate of Figure 2A: The lifespan-shortening effects of high glucose reduced by Olaparib treatment $(16 \mathrm{mM}$ Glucose). C) Biological replicate of Figure 2B: The lifespan-shortening effects of high glucose reduced by Olaparib treatment (25mM Glucose). D) Biological replicate of Figure 3A: The beneficial effect of Olaparib treatment on C. elegans lifespan in the setting of high glucose was target-specific and dependent on the PARP-1 related signaling pathway (16mM Glucose). E) Biological replicate of Figure 4A: TCF7L2 homolog, pop-1, is required for the beneficial effect in the setting of high glucose of Olaparib treatment on lifespan (Empty vector). F) Biological replicate of Figure 4B: TCF7L2 homolog, pop-1, is required for the beneficial effect in the setting of high glucose of Olaparib treatment on lifespan (pop-1 RNAi). 


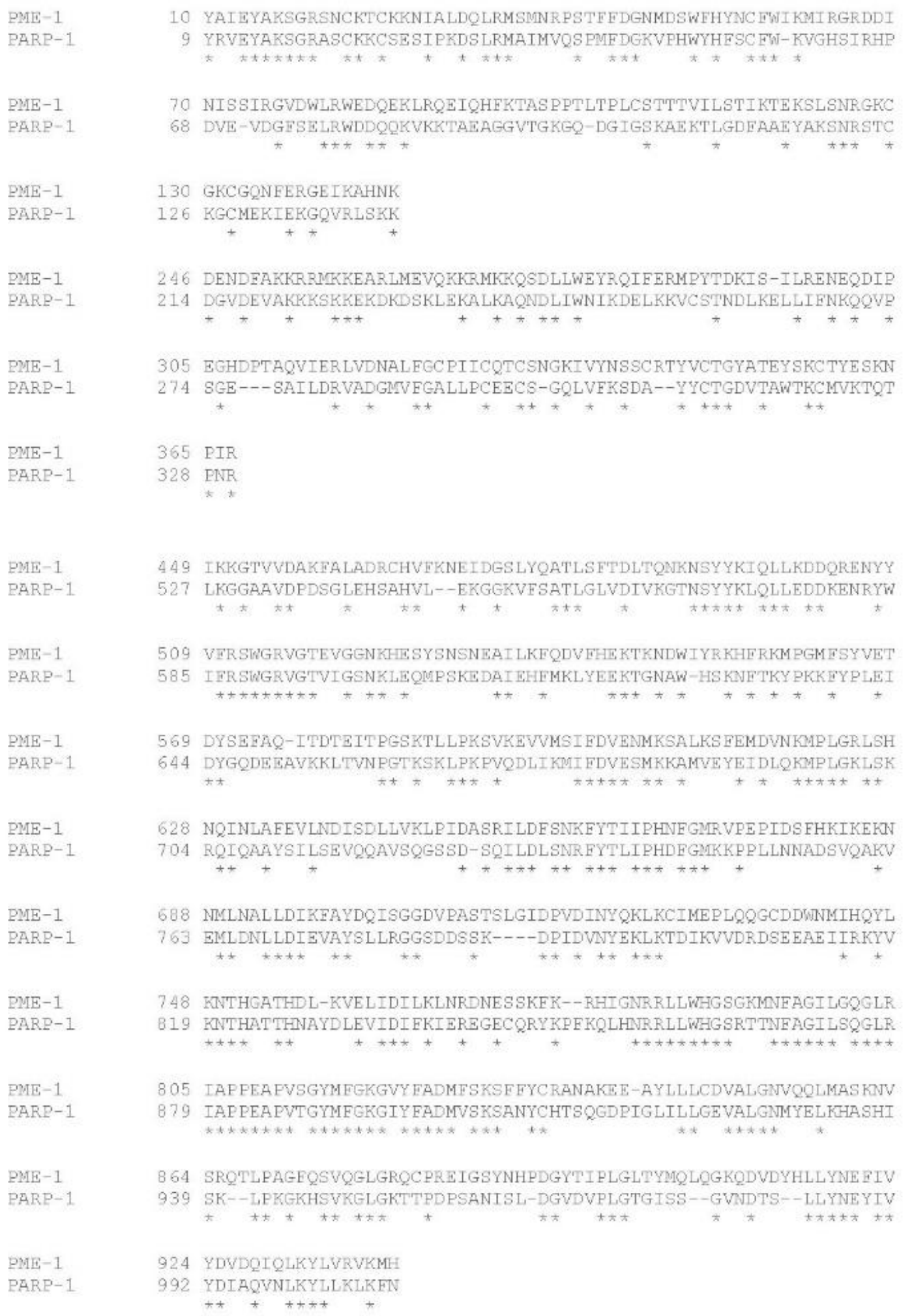

Supplementary Figure 2. Protein sequence alignment of worm pme-1 and human PARP-1. The identical residues are denoted by $*$. 


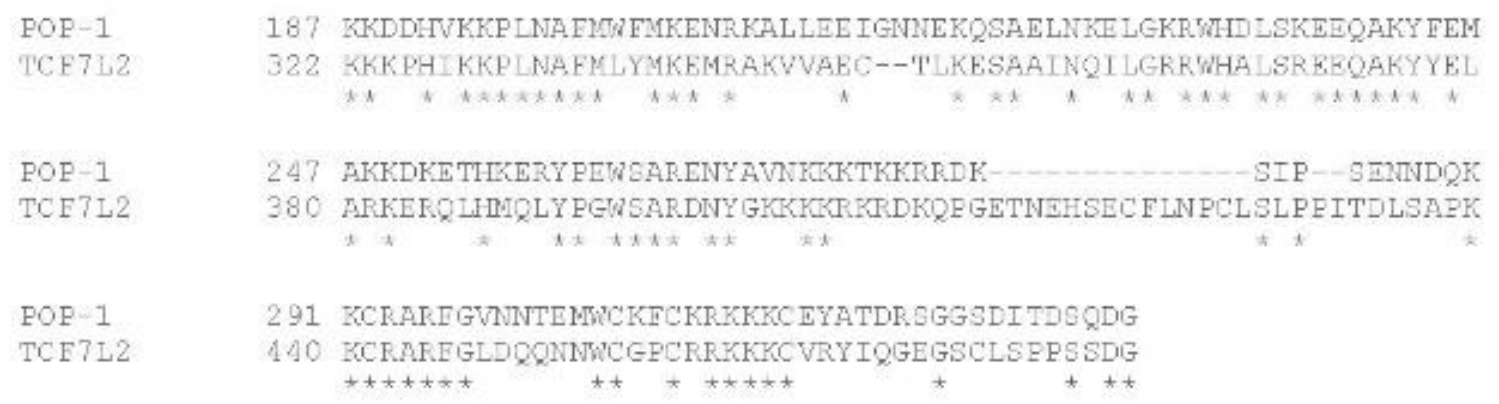

Supplementary Figure 3. Protein sequence alignment of the DNA-binding HMG box of worm pop-1 and human TCF7L2. The identical residues are denoted by *. 